\author{
GABRIELA BECKER PINTO \\ Universidad Universidad de la Empresa (UDE), Uruguay. \\ gabriela.bp@hotmail.com \\ ORCID iD: https://orcid.org/0000-0002-0879-7726 \\ JÉSSICA CRUZ DA SILVA \\ Universidad Universidad de la Empresa (UDE), Uruguay. \\ je.cruzdasilva@gmail.com \\ ORCID iD: https://orcid.org/0000-0003-083 I- I80X \\ Recibido: 30/04/2021 - Aceptado: 25/05/2021
}

Para citar este artículo / To reference this article / Para citar este artigo Becker Pinto, G. y Cruz da Silva, J. (202I). Sostenibilidad energética: el potencial de transición nuclear brasileño y el transconstitucionalismo uruguayo. Revista de Derecho, 20(39), 53-73. https://doi.org/l0.47274/DERUM/39.4

\title{
Sostenibilidad energética: el potencial de transición nuclear brasileño y el transconstitucionalismo uruguayo
}

Resumen: La presente investigación estuvo motivada por el propósito - como objetivo general del trabajo de investigación - de examinar el escenario jurídico de la Energía Nuclear entre Brasil y Uruguay bajo el sesgo del Transconstitucionalismo y Transición Energética, a partir de la convivencia analítica entre fiscalización y emprendimiento estatal- empresas propias ante el Paradigma de Sostenibilidad e Integración Regional. En el período académico, temas complementarios como la transición energética y la seguridad pública se desarrollaron de manera antagónica, denotando la necesidad de una triple hélice de acción entre tres actores principales del desarrollo: el Estado, la Academia y la Producción de Servicios a través de políticas públicas. Se concluye que el plan uruguayo buscaba ante todo impulsar la innovación conducente al desarrollo e independencia energética interna del país, logrando estratégicamente su seguridad energética con base en el atractivo político, y que el potencial exploratorio brasileño se concentra desde el momento en que se puede mitigar estigmas y pensar en el recurso nuclear como una fuente complementaria que permite satisfacer la demanda interna y una menor dependencia de las fuentes de agua.

Palabras clave: Energía nuclear, Transconstitucionalismo, Seguridad, Transición 


\title{
Sostenibilidad energética: el potencial de transición nuclear brasileño y el transconstitucionalismo uruguayo
}

\begin{abstract}
The present investigation was motivated by the purpose - as a general objective of the research work - to examine the legal scenario of Nuclear Energy between Brazil and Uruguay under the bias of Transconstitutionalism and Energy Transition, based on the analytical coexistence between inspection and State entrepreneurship before the Paradigm of Sustainability and Regional Integration. In the academic period, complementary themes such as energy transition and public security developed in an antagonistic manner, denoting the need for a triple helix of action between three main development actors: the State, the Academy, and the Production of Services through policies public. It is concluded that the Uruguayan plan sought above all to foster innovation leading to the country's internal energy development and independence, strategically achieving its energy security based on political attractiveness, and that the Brazilian exploration potential is concentrated from the moment in which that it can mitigate stigmas and think of the nuclear resource as a complementary source that makes it possible to meet domestic demand and less dependence on water sources.
\end{abstract}

Key words: Nuclear Energy, Transconstitutionalism, Security, Transition

\section{Sostenibilidad energética: el potencial de transición nuclear brasileño y el transconstitucionalismo uruguayo}

\begin{abstract}
Resumo: A presente investigação motivou-se pelo propósito - como objetivo geral do trabalho de pesquisa - de perscrutar o cenário jurídico da Energia Nuclear entre Brasil e Uruguai sob o viés do Transconstitucionalismo e da Transição Energética, a partir da coexistência analítica entre a fiscalização e o empreendedorismo Estatais perante o Paradigma da Sustentabilidade e da Integração Regional. No sopesar acadêmico, temas complementares como transição energética e segurança pública desenvolveram-se de maneira antagônica, denotando a necessidade de uma tripla hélice de atuação entre três principais atores de desenvolvimento: o Estado, a Academia, e a Produção de Serviços por intermédio de políticas públicas. Conclui-se que o plano Uruguaio procurou sobretudo fomentar a inovação acarretando o desenvolvimento energético interno e a independência do país, alcançando de forma estratégica sua segurança energética pautada no atrativo político, e que o potencial de exploração Brasileiro concentra-se a partir do momento em que este conseguir mitigar estigmas e pensar o recurso nuclear como fonte complementar que possibilite o atendimento à demanda interna e a menor dependência de fontes hídricas.
\end{abstract}

Palavras-chave: Energia Nuclear, Transconstitucionalismo, Segurança, Transição 


\section{Introducción}

Frente a un período de grandes turbulencias políticas, económicas e incluso de desafíos sociales que se han producido en los últimos años, hemos visto importantes debates sobre la agenda internacional que se han convertido en objetivos para la priorización de las naciones. El desarrollo sostenible y sus implicaciones para el sector energético siguen encabezando la lista, debido a la gran carga sobre el medio ambiente y los constantes cambios climáticos derivados del modelo económico en el que vivimos. La perspectiva de un futuro sostenible con menos emisiones de contaminantes a la atmósfera nos hace cuestionar las formas y tipologías para llegar a un modelo de desarrollo, que utilice energías que nos permitan brindar niveles satisfactorios de calidad de vida a las próximas generaciones.

Pensando en este recurso, surge la tan temida e incomprendida energía nuclear, que, si bien es una de las principales fuentes de energía del mundo y se extrae a través de la ruptura del átomo de uranio, tiene un acalorado debate en torno a los medios - las plantas y sus Riesgos más allá de los umbrales del debate sobre energía y seguridad nacional - y propósitos de su uso - pacíficos o no. Ciertamente, cualquier fuente de energía puede presentar sus dualidades en cuanto a su uso, sus finalidades y sus posibles implicaciones en el ámbito jurídico y de seguridad, ya sea en un ámbito más amplio - la seguridad internacional, o en un ámbito más intrínseco - la seguridad energética.

Se propone, entonces, cuestionar los aspectos legales y constitucionales que se pueden utilizar o incluso que ya forman parte del plan de desarrollo de la energía nuclear en Brasil, considerando que, si bien es una fuente no renovable, el uranio es parte de una de las riquezas ofrecidas en suelo brasileño, con un gran potencial para asumir el papel de energía complementaria a las fuentes renovables, además de ser una opción para reducir, en términos relativos, el uso de fuentes fósiles.

Sin embargo, la propuesta es utilizar como modelo, al menos a efectos de inspiración, el caso del vecino sudamericano, Uruguay, aunque menos en su número de habitantes y en temas territoriales, puede enseñarnos en grandes materias, principalmente, cuando buscamos temas específicos y pudimos extraer buenas prácticas de aplicación y el potencial que aún se puede explorar en términos de crecimiento, desarrollo tecnológico e innovación en materia de bajas emisiones de carbono.

Sin embargo, la premisa rectora de este trabajo fue que se asumiría que las estrategias delineadas en el Plan Energético de Uruguay (PE 2005-2030), que guiaron a Uruguay hacia el 'BIG PUSH', podrían servir de modelo como forma de ayudar para esbozar impulsos o incluso inspirar a Brasil en el desarrollo de la Energía Nuclear, como factor exponencial, no solo de transición sino también de seguridad energética.

La metodología utilizada en este trabajo será cualitativa, en la que se decidió buscar fuentes no exhaustivas del proceso de transición de ambos países, teniendo en cuenta los aspectos territoriales, geopolíticos y tecnológicos involucrados. Para ello se realizaron investigaciones, relevamientos y análisis de textos, artículos, proyectos de reforma constitucional, legislación, Políticas Energéticas y sus estrategias, además de documentos oficiales tanto de gobiernos, entidades, organismos y agencias internacionales. El enfoque de este proyecto se basa en contenido histórico, concentrado en el período 2015 - 2020, aunque los Planes de Energía están proyectados hasta el año 2050. 
Se observa que, de ninguna manera nuestra intención de comparar ambos países es incomparable en características y culturas, sin embargo, aunque diferente en innumerables aspectos, se cree que el modelo uruguayo, que contiene los lineamientos y estrategias delineados en el Plan Energético de Uruguay 2005 - 2030, con el que diseñó espléndidamente su camino hacia la transición energética utilizando energías renovables, además del potencial de mercado y entorno empresarial, avanzó hacia una economía baja en carbono (low carbon) y pleno desarrollo y concienciación de su población y debería ser utilizado como una fuente inspiradora o al menos visto como un puente a recorrer en términos estratégicos por Brasil con respecto a la adopción de la Energía Nuclear como fuente de la transición.

\section{La energía nuclear como fuente de energía limpia}

\section{1. ¿Qué es la energía nuclear?}

La Energía Nuclear se puede definir como la energía liberada de reacciones en cadena que se desarrollan en el núcleo de los átomos y que se convierten en energía eléctrica, ya sea por fisión o fusión de radioisótopos. (LEHTO, 2007). Así, los radioisótopos pueden denominarse como el conjunto de átomos con núcleos inestables que implican emisión de radiación (BRASIL, 2018). Estas radiaciones ocurren a través de estas fisiones o fusiones nucleares, que son básicamente procesos de liberación de energía química. Sin embargo, la fisión consiste en la fisión de un radioisótopo utilizando neutrones libres. La fusión, por otro lado, resulta de la unión de átomos. Así, en ambos procesos, surge un nuevo elemento químico que genera un nivel masivo de energía (BRAGA, 2019a). Por tanto, este conglomerado fenomenológico posterior a la desintegración nuclear, debido a la inestabilidad presente en el núcleo de estos átomos, da como resultado la producción de partículas (alfa y beta) o radiación (gamma) definiendo lo que se puede conceptualizar como radiactividad.

El conjunto de fuentes utilizadas para crear energía en un locus territorial dado puede denominarse matriz energética (ENERGÉTICA, 2019). Las matrices energéticas están reguladas por normas especiales, que prevén la conducta de las personas naturales y jurídicas en relación con determinadas actividades que impliquen manipulación y/o exposición a materiales y productos radiactivos, ya sean de origen natural o procesado (BRAGA, 2019a). Es este conjunto de reglas el que configura el campo de acción de la denominada Ley Nuclear.

Así, la energía nuclear realiza el trabajo de una fuerza y puede verse con un conjunto de factores, es decir, además de los energéticos, económicos y ecológicos. Según Naciones Unidas, el desarrollo sostenible está vinculado a la satisfacción de las necesidades del presente sin el compromiso de las generaciones futuras (ORGANIZATION, 2019). Ya que, según (CRUZ; BODNAR; FERRER, 2012), la sustentabilidad consiste en un paradigma metajurídico que guía la sociedad global y la actuación del intérprete del Derecho en el tercer milenio. Por eso la figura del transconstitucionalismo en este campo es tan importante, ya que permea soluciones a problemas jurídico-constitucionales que se presentan simultáneamente a diferentes órdenes. Por tanto, los deseos perseguidos en esta transición energética se ennoblecen. 


\subsection{El contexto de la transición energética}

El agotamiento y la sobrecarga del medio ambiente, producto del actual modelo de desarrollo mundial y derivado de las más diversas actividades económicas, principalmente en el aspecto de exploración de recursos, son solo las "caras" derivadas de la emisión de GEI (Gases de Efecto Invernadero) como Reflejo de esto, preocupaciones alarmantes sobre el calentamiento global ya están mostrando sus rasgos en eventos y eventos climáticos extremos y cada vez más frecuentes (REIS, 2015).

En consecuencia, las discusiones sobre el medio ambiente y los tipos de recursos para la generación de energía que componen la matriz energética mundial, asumen un peso ascendente en la agenda política de los Estados, especialmente después de la década del 90. Contrario a esta explosión sostenible, encontramos, aunque de manera desproporcionada, la dependencia de estos mismos Estados (SANTOS, 2016) de los sectores energéticos, los cuales se apoyan firmemente en la explotación de combustibles fósiles, que a su vez generan profusos impactos al agotamiento ambiental. Además de este choque, se debe tener en cuenta el rol de nuevos actores que generan un cambio drástico, no solo en la forma en que se explotan los recursos naturales sino sobre todo en la forma de participación de los consumidores y competitividad en el sector energético para el mundo y nuevas formas de generación.

Por tanto, en este escenario se presenta una nueva fase de la transición energética, construyendo y contribuyendo a nuevos patrones de consumo que implican en procesos tecnológicos más avanzados y que cumplen esencialmente la Era de la transición, teniendo como principal motor los cambios climáticos que conducen a una desarrollo económico más sostenible.

La transición energética que estamos atravesando en este momento es el resultado de otras versiones que hemos ido atravesando a lo largo de los siglos, con ello una transmutación significativa del paradigma energético geopolítico vivido hasta el momento.

Asistimos a una profunda transición energética, tanto para los escenarios de oferta y demanda, en la forma de energía que llega a los consumidores, como para los factores que determinan la influencia y las relaciones de poder entre naciones y regiones. [...] La transición energética que vivimos hoy, bajo diferentes formas y ritmos, tiene unas características inéditas. Es el primero con el clima como impulso motivador. Necesariamente tendrá que ser más intenso y más rápido que las transiciones anteriores, si queremos lograr los objetivos establecidos en 2015 en la Conferencia de las Naciones Unidas sobre el Cambio Climático en París. (Camargo, 2020, p.10).

En este contexto, la transición energética puede entenderse como un cambio de paradoja. Si antes la primacía energética se basaba en un contexto esencialmente económico, hoy se nos lleva a repensar todo este modelo de desarrollo y reformularlo. Si bien hemos sido testigos de varias fases de la transición desde las Revoluciones Industriales I y II, que hoy presenciamos, nos lleva directamente a una nueva visión, cuya preocupación es esencialmente ambiental y sustentable. 


\subsection{La energía nuclear como fuente de energía limpia y potencial para la transición energética}

La perspectiva de un futuro sostenible con menos emisiones de contaminantes a la atmósfera nos hace cuestionar las formas y tipologías para llegar a un modelo de desarrollo, que utilice energías que nos permitan brindar niveles satisfactorios de calidad de vida a las próximas generaciones.

Así, autores y varios técnicos del área reconocen a la energía nuclear como una poderosa fuente capaz de cubrir necesidades en una matriz energética, siendo considerada, por tanto, una fuente limpia, especialmente por emitir índices de carbono ínfimos en la atmósfera. En este sentido, el OIEA (Organismo Internacional de Energía Atómica), destaca que:

La energía nuclear ya aporta alrededor del 10\% de la electricidad mundial, pero contribuye con casi el 30\% de toda la matriz eléctrica baja en carbono. La energía nuclear seguirá siendo esencial para lograr un futuro con bajas emisiones de carbono, que los líderes mundiales acuerdan perseguir. (OIEA, 2020, p.1) (Nuestra traducción).

Desde este punto de vista, la ABEN (Asociación Brasileña de Energía Nuclear), reitera el pensamiento de que esta fuente de energía no solo contribuye fuertemente al avance de las medidas para lograr menores niveles de emisiones de carbono, sino que además considera que el desarrollo de la energía nuclear es parte de la forma comprobada de crear energía asequible y con bajas emisiones de carbono, así como de crear empleos y cadenas de suministro locales de alto valor a largo plazo. (ABEN, 2020).

Adicionalmente, por su capacidad y alta densidad energética en potencia instalada (MW) por área reducida y ocupada, las centrales nucleares pueden instalarse relativamente cerca de los núcleos urbanos, lo que conlleva a una mayor preservación de áreas sensibles y hacen de este recurso la principal fuente de energía. generación con reducción de impactos ambientales y sociales significativos, incluso proporcionando el avance local del espacio de su instalación. Por tanto, la adopción de la energía nuclear como fuente generadora y participante activo en la matriz energética global, puede verse como una estrategia de amplificación y desarrollo nacional, además de, por supuesto, altamente sostenible.

Por ello, el papel de la energía nuclear puede entenderse como motor de la transición y un nuevo enfoque a pensar, no solo en términos de generación, sino como un nuevo horizonte que emerge para el desarrollo económico sostenible, basado en nuevos tecnologías destinadas específicamente para el sesgo bajo en carbono. Además, el recurso extraído del uranio puede considerarse una fuente propulsora que permite mejorar el proceso de quema de átomos y aumentar los estándares de seguridad.

En definitiva, la aplicación de este mineral como recurso energético natural, si se idealiza como puente inspirador entre el cruce del calentamiento global y la escasez de recursos para el desarrollo sostenible, servirá de estímulo para la conformación de nuevas alternativas y complementarias, limpias. y bases consistentes que posibiliten no solo el proyecto global bajo en carbono, sino que también promuevan la promoción de la innovación, la competitividad y el surgimiento de nuevos actores en el mercado, 
capaces de conducir a una reformulación del sistema, especialmente en los aspectos tecnológicos, geopolíticos y relacionados con la búsqueda de la seguridad energética.

\section{Energía Nuclear en Brasil}

\subsection{Energía nuclear en Brasil y su contexto histórico}

Las décadas que siguieron a 1930 fueron sumamente importantes para el desarrollo de la física brasileña moderna, ya que albergaría importantes eventos, entre ellos la creación de la Facultad de Filosofía, Ciencias y Letras de la Universidad de São Paulo, y la exportación secreta y el sistema. de arenas monazíticas para Estados Unidos (DE BIASI, 1979), lo cual corroboró en un ambiente cooperativo en relación al Proyecto Manhattan de ese país, el cual, fue lanzado en la responsabilidad de la construcción de una bomba atómica que utilizaría los desarrollos en la tecnología de la reproducción de Fisión nuclear durante la Segunda Guerra Mundial.

En este contexto, el debate sobre el posible uso y preservación de sus propios recursos naturales para la producción de energía atómica y la elaboración de proyectos oficiales se inició en Brasil a mediados de 1945. Así, proveedor de materias primas a Estados Unidos, el país suministró hasta la década de 1950 abundantes cantidades de minerales atómicos de los que es rico. Y, con la implementación del principio de compensación específica, por cada exportación de minerales estratégicos, se produjo una transferencia de tecnología útil destinada a mejorar el sector nuclear nacional (INGULSTAD; LIXINSKI, 2013).

El estudio de Dimis da Costa Braga (2019) es muy oportuno cuando señala que, ante la demostración de la dificultad para obtener tecnología en el exterior, el país buscó seguir los avances internacionales en el sector. Y, por tanto, dejó de buscar exclusivamente la adquisición de tecnología autóctona del exterior, ya sea para la producción de uranio metálico, bien, para el desarrollo de su enriquecimiento (2019b, s.p.).

Porque, fue en el contexto del uso bélico de la tecnología nuclear que Brasil buscó establecer los primeros pasos hacia la creación de un centro espacial brasileño y, a fines de la década de 1960, este centro pasó a llamarse INPE (Instituto Nacional de Investigaciones Espaciales), cuyo El objetivo principal fue diseñar y ejecutar la construcción de un cohete lanzador de satélites (BRAGA, 2019b).

Como no había ninguna industria que desarrollara o aplicara los resultados de estas investigaciones realizadas en el INPE y otras agencias hasta entonces, según una retrospectiva brasileña dilucidada por estudiosos, en 1951 la creación del CNPq (Conselho Nacional de Desenvolvimento Científico e Tecnológico), y A propuesta de este Consejo, entraron en escena varios otros centros de investigación dedicados exclusivamente a la física nuclear. Entre ellos, el rigor de la época es evidente, el IEA (Instituto de Energía Atómica), ubicado en el estado de São Paulo y el IPR (Instituto de Investigaciones Radiactivas) instalado junto a la Universidad Federal de Minas Gerais, en el estado de Minas Gerais, ambos en el período de 1953. En 1960 se crea el Laboratorio de Dosimetría, transformado posteriormente, en 1971, en el Instituto de Radioprotección y Dosimetría. En los años siguientes, en 1963, se creó el IEN (Instituto de Ingeniería Nuclear), en el estado de Río de Janeiro, y, finalmente, este ciclo culminó con la institución de CBTN 
(Companhia Brasileira de Tecnologia Nuclear), que incorpora el IPR de Minas Gerais, que luego se denominaría CDTN (Centro de Desarrollo Tecnológico Nuclear), vinculado al CNEN, con la Universidad Federal de Minas Gerais, en el estado de Minas Gerais. Es crucial reflexionar que, en este momento, por lo tanto, el locus territorial nuclear brasileño ha superado una expansión considerable (DE BIASI, 1979).

Con la adquisición de 03 (tres) centrifugadoras de Alemania, interceptadas por tropas de ocupación inglesas a pedido del gobierno estadounidense y solo liberadas después de demostrar que enriquecerían muy pocas cantidades de uranio y, con honorarios mínimos (BRAGA, 2019b), se llevaron a cabo intereses académicos en junto con acciones políticas estratégicas para apalancar el sector atómico brasileño.

Según el esquema histórico de la época, los propósitos nucleares no eran exclusivamente pacíficos en el país, ni tampoco en otras naciones. Así, en un contexto de combate al carácter clandestino de las operaciones de Brasil en el exterior, considerando la obligación de que todos los compromisos en materia nuclear pasen al escrutinio del Congreso Nacional, y de suspensión a las exportaciones de uranio, torio, compuestos, minerales u otro país estratégico. material, se constituyó CNEN (Comisión Nacional de Energía Nuclear) (GIROTTI, 1984).

Asimismo, según Dimis da Costa Braga (2019), se creía que la Energía Nuclear por proceso de fisión llegaría a jugar, ya a mediados de la década de 1970, un papel preponderante en la generación de electricidad global, y se dieron grandes pasos en el sentido de expandirla. (2019a, s.p.) Sin embargo, posterior a este optimismo nuclear, emergen las poblaciones de los países más desarrollados y democráticos, una desconfianza simultánea tangente a los peligros derivados de las actividades nucleares (MACHADO, 1980).

Como una forma de garantizar la seguridad, proteger el medio ambiente, participar en los procesos de toma de decisiones e instituir salvaguardias efectivas para las actividades nucleares a escala global, nació el OIEA (BRAGA, 2019b).

\subsection{Programa nuclear brasileño}

Las bases del Programa Nuclear Brasileño se establecieron durante el período del régimen de gobierno militar, adornado por temas sensibles y estratégicos. El Programa, se enfatiza, tenía negocios en el país con Estados Unidos y, de manera secuencial, con el Acuerdo Nuclear Brasil - Alemania.

Firmado en junio de 1975, el Acuerdo Brasil - Alemania preveía la instalación de 08 (ocho) centrales nucleares, la creación de empresas de prospección y exploración de uranio, fabricación de elementos combustibles, fabricación de componentes pesados para plantas y reactores, reprocesamiento de combustible irradiado, enriquecimiento de uranio, y, toda la creación de una verdadera industria nuclear (BRAGA, 2019b). Sin embargo, se estigmatizó por dar cuenta de una pérdida económica con un costo natural superior al del sector privado en el país, subvencionado por la nueva industria nuclear.

Aunque Brasil sufrió los boicots y los intereses económicos y estratégicos de estas naciones, la historia ha demostrado que incluso siguiendo caminos tan incómodos, la nación finalmente detuvo el ciclo completo del combustible nuclear. Y, por cierto, 
sucedió no solo con el desarrollo de su propia tecnología, sino también con la firma de estos acuerdos internacionales.

A partir de la década del 90, el Programa sufrió flexibilidades y fue desmantelado, lo que le permitió distanciarse, en la perspectiva de la integración económica y energética con los países del MERCOSUR (Mercado Común del Sur). Entre 1991 y 1994, el país se adhirió a regímenes internacionales de no proliferación nuclear, aceptó amplias salvaguardias internacionales, incluidas las del OIEA, y le siguió un componente del área latinoamericana libre de armas nucleares (PATTI, 2014).

\subsection{Plantas de energía nuclear de Angra}

El paradigma que se creó alrededor de las centrales nucleares brasileñas proviene de los estigmas utilizados dentro del propio Programa Nuclear Brasileño. Esta última, que inicialmente contemplaba la elaboración de 08 (ocho) centrales nucleares (BRANDÃO, 2008), en cuanto se quedara con una sostenibilidad económica afectada por las perturbaciones ocurridas en las primeras edificaciones.

La construcción de la Central Nuclear Angra I, en el estado de Río de Janeiro, marcó la primera fase del Programa y obtuvo la licencia para iniciar operaciones en diciembre de 1984.

Sin embargo, sufrió varias interrupciones, incluso por orden judicial y fue inaugurado oficialmente en 1985. Con un reactor PWR (reactor de agua a presión), tecnología suministrada por la empresa estadounidense Westinghouse, cuenta con 657 MW de potencia instalada. Sin embargo, desde el preámbulo de las operaciones de Angra I, hubo frecuentes fugas de material radiactivo a través de las varillas que almacenan el combustible fósil dentro del reactor nuclear y fallas en el manejo del material de la Planta (BERMANN, 2002).

El otorgamiento de la licencia para la construcción de la Central Nuclear Angra II se dio en 1981, sin embargo, un largo lapso la separó de su inauguración oficial. La licencia para iniciar operaciones no se otorgó hasta marzo de 2000, pero fue en el período de julio de 2011 cuando la Planta finalmente logró iniciar sus actividades. Con un reactor PWR, tecnología suministrada por la empresa alemana KWU, presenta $1.350 \mathrm{KW}$ de potencia neta (PATTI, 2014).

El programa nuclear se revitalizó a principios de la década de 2000. En 1998, el Congreso aprobó la reactivación de proyectos para la construcción de una planta industrial que tenía como objetivo producir combustible nuclear y alentó la colaboración entre los centros de investigación de la Armada y la industria nuclear. Se racionalizó la gestión de la Central Nuclear Angra I y las posteriores y surgió una nueva empresa pública denominada Eletronuclear de la fusión de Furnas y Nuclen, siendo filial de Eletrobrás.

Por tanto, quedó definitivamente constituido el proyecto de construcción de la Central Nuclear de Angra III. Sin embargo, debido al escenario nacional, aún no ha terminado. Pero de una forma u otra, es innegable el triunfo de que Brasil tenga dos Plantas Nucleares muy eficientes que son consideradas patrimonio nacional, ya que logró conquistar el dominio de la tecnología del uranio enriquecido. 


\section{La energía nuclear en Brasil y Uruguay desde el punto de vista legal y de seguridad}

\subsection{Ley constitucional brasileña}

El sistema legal brasileño otorga un rol de centralidad constitucional en materia energética, en particular, el tratamiento que le da la legislatura nacional a las actividades nucleares. Se puede decir, en este diapasón, que la ley ha sido el mecanismo utilizado para mantener el control estatal sobre estas operaciones y la dependencia del sector eléctrico para el financiamiento público (BRAGA, 2019a).

La primera disposición expresada en materia nuclear está contenida en la Enmienda Constitucional núm. 1/1969 a la Constitución de 1967, inmediatamente en su artículo 8, inciso XVII: "Compete à União: [...] XVII - legislar sobre: [...] i) águas, telecomunicações, serviço postal e energia (elétrica, térmica, nuclear ou qualquer outra)." (BRASIL, 1969, s.p.) El Subcomité Temático de Salud, Seguridad y Medio Ambiente emitió instrucciones normativas en su artículo 70, en el que la opinión del relator tendía a restringir el uso de Energía Nuclear en el territorio nacional: "Proíbe-se no Território Nacional a instalação e funcionamento de reatores nucleares, exceto para finalidades Cientificas. [...] As demais atividades nucleares serão exercidas mediante controle do Poder Público, assegurandose a fiscalização supletiva pelas entidades representativas da sociedade civil." (BRAGA, 2019a)

Según Dimis da Costa Braga (2019), no se puede negar que el marco democrático de 1988 estableció muchos preceptos encaminados a garantizar la máxima participación de la sociedad en términos de deliberación participativa en el proceso de toma de decisiones en materia de generación eléctrica. (2019, s.p.) El artículo 225 de la Carta Magna es oportuno cuando establece el medio ambiente como derecho y principio fundamental, requiriendo en su inciso IV, previo estudio preliminar de impacto ambiental y amplia publicidad para la instalación de obras o actividades que potencialmente causen degradación del medio ambiente.

En relación a la explotación de fuentes de energía, gran parte de estas estaban abiertas a la acción tanto del Estado como de entidades privadas. Lo que se puede observar con el artículo 21 de la Constitución Federal de 1988, "Compete à União: XII - explorar, diretamente ou mediante autorização, concessão ou permissão: [...]b) os serviços e instalaçóes de energia elétrica e o aproveitamento energético dos cursos de água, em articulação com os Estados onde se situam os potenciais hidroenergéticos;" (BRASIL, 1988)

Sin embargo, es interesante notar que en el ámbito nuclear, a diferencia de los demás, quedó relegado a la dependencia del Estado. Su empleo fue restringido y colocado en condición centralizada en la Unión, dependiente del financiamiento estatal y condicionado a la expansión del sector a la autorización del Poder Legislativo, según lo dispuesto en los artículos 21 y 22 de la Constitución Federal de 1988:

Compete à União: [...] XXIII - explorar os serviços e instalaçõos nucleares de qualquer natureza e exercer monopólio estatal sobre a pesquisa, a lavra, o enriquecimento e reprocessamento, a industrialização e o comércio de minérios nucleares e seus derivados, atendidos os seguintes princípios e condições: a) toda atividade nuclear em território nacional somente será admitida para fins pacíficos e mediante aprovação do 
Congresso Nacional; b) sob regime de permissão, são autorizadas a comercialização e a utilização de Radioisótopos para a pesquisa e usos médicos, agrícolas e industriais; (Redação dada pela Emenda Constitucional no 49, de 2006) c) sob regime de permissão, são autorizadas a produção, comercialização e utilização de Radioisótopos de meiavida igual ou inferior a duas horas; (Redação dada pela Emenda Constitucional no 49, de 2006) d) a responsabilidade civil por danos nucleares independe da existência de culpa; (Redação dada pela Emenda Constitucional no 49, de 2006). (BRASIL, 1988)

La Carta Fundamental es, por tanto, competencia exclusiva de la Unión para la gestión y el desarrollo de las actividades nucleares en todas sus fases, incluida la legislación. Esta rigidez constitucional es importante y continúa estableciendo la competencia exclusiva del Congreso Nacional para aprobar también iniciativas del Poder Ejecutivo. Sin embargo, el temor reside en esta nube de ignorancia en el campo nuclear brasileño, que puede desechar la opción termonuclear sin ponderar otros debates e instituciones sociales.

El propio rol que actualmente ocupa la CNEN como máximo órgano rector en el manejo nuclear debe ser visto con cautela, una vez delegada la competencia del Congreso Nacional, ha adquirido tal potestad con la exclusiva responsabilidad de inspección, planificación, supervisión, investigación científica e instrucción normativa nacional del sector. Sin embargo, es innegable que la actualización normativa es de suma importancia en este escenario y que las leyes infraconstitucionales continúan dictando las grandes reservas y urgencias en el contexto legal nuclear.

Uno de los grandes aspectos a discutir en el ámbito nuclear es la priorización de la investigación regulatoria dentro de una agencia reguladora como la CNEN. A partir de estas premisas, pueden surgir caminos más eficientes y democráticos en el campo energético y fortalecer el Estado de Derecho Democrático en su conjunto. (HEILBRON; COSTA, 2017)

\subsection{Transconstitucionalismo uruguayo}

El uso de energía nuclear en Uruguay está prohibido por la Ley n. 16.832, promulgada en 1997, que regula la actualización del Sistema Eléctrico Nacional y crea la Unidad Reguladora de Energía (HONTY, 2011). La presente orden prevé la prohibición en su artículo 27 y destaca que ningún agente del mercado eléctrico mayorista podrá suscribir contratos de suministro eléctrico utilizando generadores nucleares o generadores extranjeros, ya que las plantas contaminarían el territorio nacional.

A pesar de la comprensión legislativa contemporánea, el país se basó en numerosos esfuerzos institucionales y normativos para estructurar el sector nuclear. La creación de la Autoridad Nacional Reguladora de Radioprotección, a través de la Ley n. 17.930, 2005; la Comisión Nacional de Energía Atómica y la Dirección Nacional de Tecnología Nuclear, mediante Ley núm. 15.809, 1986; el Centro de Investigadores Nucleares, en 1966; y, la concesión de una prestación de jubilación a personas que realizaban actividades laborales expuestas a radiación de rayos $\mathrm{X}$ o manipulación de elementos radiactivos son ejemplos iniciales.

Finalmente, en 1964, se compró un pequeño reactor nuclear a los Estados Unidos para investigación y entrenamiento personal. Sin embargo, a través de la dialéctica construida 
por los accidentes de Chernobyl (Ucrania), Goiânia y cesio-137 (Brasil), y Fukushima I (Japón), se encendieron los debates en el ámbito político y social uruguayo (HONTY, 2011). Mientras el gobierno iniciaba una fase de evaluación de la energía nuclear, se contrataron especialistas para consultar a la población y evaluar los recursos humanos y la tecnología disponible. Con la creciente inversión en energías renovables (eólica, biomasa, solar fotovoltaica, termosolar, hidráulica y biocombustibles) y la exploración de la presencia de petróleo en el país, el debate nuclear pasó a un segundo plano y bajo un nuevo paradigma de sostenibilidad y uso pacífico.

Si bien los argumentos en contra del uso nuclear se superponen en la monetización exhaustiva y los requisitos de seguridad internacional, plantea factores importantes para la descarbonización de la generación eléctrica, ya que se combina con tecnologías renovables intermitentes con facilidad, ya que no dependen de las condiciones climáticas y generan energía en todo momento.

El análisis de alto desempeño energético en Uruguay considera tres factores importantes: el desempeño y liderazgo de las empresas públicas como generadoras de servicios, la cultura uruguaya de respeto al medio ambiente y el entorno regulatorio. La materialización de la idea de ciudades inteligentes proviene de un buen desempeño en la generación de servicios. (PRESUPUESTO, 2019)

Este estudio de probabilidades también destaca la importancia del Estado para orientar y brindar bienestar a los ciudadanos a través de políticas públicas orientadas a la calidad de vida, y no a favorecer decisiones coyunturales. Es decir, aunque sea tenue, debe existir un límite entre el Estado que supervisa y el mismo Estado que asume.

El sector energético uruguayo ha experimentado varios cambios en los últimos años, la energía se ha convertido en un peso enorme en las cuentas nacionales, ya que su consumo se ha incrementado exponencialmente. Esto también generó una gran preocupación en el escenario normativo, cuya legislación se preocupó por establecer un uso más racional y sostenible de estas energías. Un gran ejemplo de esto es la institución de la Ley de Eficiencia Energética n. 18.597, 2009 y la Política Energética acordada entre todos los partidos políticos (PEDROUZO, 2014).

Actualmente, la eficiencia es definida por la doctrina administrativa uruguaya como la proporcionalidad razonable entre la actividad realizada y los medios empleados en relación a los resultados perseguidos y obtenidos, siendo una traducción del principio de arraigo constitucional. La ley, por tanto, ha buscado reflejarse en un instrumento de impacto a nivel de política energética, económica y medioambiental. De esta manera, ha estructurado y regulado un sistema de actores estatales y no estatales con diferentes grados de responsabilidad, obligaciones y restricciones para proteger el interés común.

El análisis sobre la regulación de la institucionalidad normativa del sector energético es importante para determinar si se enfrenta a una norma de actuación (jerarquía normativa inferior - referirse a principios en sentido estricto y las reglas) o una norma final (jerarquía normativa superior - referirse a la Constitución y la ley), y comprender cuál es el margen de discrecionalidad con el que la Administración tiene para dictar sus decisiones.

Como diría el reconocido jurista uruguayo Héctor Gros Espiell, “No se puede describir realísticamente un sistema social, sin referirse al orden jurídico aplicable a él. Por eso ha 
dicho con toda razón recientemente Georges Burdeau; 'en voulant demeurer juristes, nous faisons preuve de réalisme'”. (ESPIELL, 1968, p. 15)

\subsection{Los desafios de la energía nuclear como cuestión de seguridad pública}

Así, como señala el autor brasileño Guilherme de Souza Nucci (2016), en su obra 'Direitos Humanos versus Segurança Pública', los derechos humanos son inherentes a la naturaleza humana y confieren una individualidad real al ser para el logro de condiciones dignas de supervivencia, y de esa manera. manera en que están abrazados por el manto de la ley natural y también positiva. La dignidad humana, por tanto, se entrelaza aquí y corresponderá al Estado proteger tanto al individuo en lo que respecta a la vida, la libertad, la dignidad y, en especial, el orden público y la seguridad. Tales condiciones, aunque específicas, son inseparables en su conjunto y tienen como objetivo proteger al individuo incluso de las acciones del propio Estado. (2016, s.p.)

La seguridad pública, generalmente vista con un sesgo antagónico a los derechos humanos, necesita interactuar con estos derechos. Si bien es un deber del Estado, también es una obligación comunitaria. La seguridad pública, al ser vista como un derecho común de todos y como una política pública, por lo tanto, debe ser perseguida por toda la comunidad. Dado que la concentración entre los cuerpos policiales estatales, el Ministerio Público y el Poder Judicial para este fin es fundamental.

\subsection{Seguridad energética y sus desafios}

A pesar de los desafíos observados en los campos legal y de seguridad pública, se agrega a la discusión un tema ampliamente discutido llamado Seguridad Energética.

Este término, aunque consagrado por la OIEA, aún tiene discusiones sobre parámetros y sobre todo la amplitud de su concepto, muchas veces enfrentándose al término de seguridad internacional - que nos lleva a un orden jurídico global - o incluso como una extensión de ella.

Creada básicamente como un mecanismo de protección para los países consumidores de energía, que dependían del petróleo suministrado por la OPEP (Organización Mundial del Petróleo), la OIEA instituyó el concepto de Seguridad Energética, definiéndolo como la disponibilidad ininterrumpida de fuentes de energía a un precio asequible. Este término se solidificó en un momento en que el mundo acababa de atravesar otra fase de transición energética, del carbón al petróleo, y principalmente, con la crisis del petróleo que siguió. El Organismo aún segmenta este concepto en dos aspectos: largo y corto plazo.

La seguridad energética tiene muchos aspectos: la seguridad energética a largo plazo se trata principalmente de inversiones oportunas para suministrar energía de acuerdo con los desarrollos económicos y las necesidades ambientales. Por otro lado, la seguridad energética a corto plazo se centra en la capacidad del sistema eléctrico para reaccionar rápidamente ante cambios repentinos en el equilibrio entre oferta y demanda. (OIEA, 1973 s.p., nuestra traducción). 
En el primer aspecto, se asume la seguridad energética cuando las inversiones logran seguir la evolución de las demandas ambientales, es decir, seguir los procesos en los que es necesario llevar a cabo el proceso de transición energética y, principalmente, el desarrollo económico del país. país. Mientras que, el segundo aspecto, se centra en la capacidad del Estado para reaccionar en el suministro de energía doméstica aun cuando existan desequilibrios, cambios y afluencias a la oferta y demanda nacional.

Sin embargo, según el autor Alexandre Nina (2020), el desafío de la Seguridad Energética sienta precedentes y complejidades más allá de lo esperado, en el que, por "disponibilidad ininterrumpida", es posible comprender posibles interrupciones en el suministro y pueden ser causas de innumerables variables, intencionales o no, que afecten a la infraestructura energética, como los eventos climáticos. Por tanto, de este contexto de complejidad del término y entendimiento colectivo de la seguridad energética, surge un nuevo enfoque para la mitigación de riesgos, incorporando al concepto, indicadores que permiten "visualizar", en términos más prácticos, el avance o amenaza, a la seguridad. vulnerabilidad de los países, que aquí destacamos como: las tasas de diversificación de la matriz energética; la relación entre importación y demanda interna; la capacidad de utilizar fuentes alternativas disponibles en lugar de depender de otras fuentes; o incluso la capacidad de desarrollar planes estratégicos que apunten a minimizar la tasa de interdependencia energética. (2020, n.p.)

\section{Perspectivas de la energía nuclear en Brasil y el modelo uruguayo de transición y seguridad energética}

\subsection{Perspectivas nucleares como factor potencial para la transición y seguridad energética brasileñas}

La matriz energética brasileña es considerada una de las más diversificadas del mundo y cuenta con abundantes y completamente diversos recursos naturales en su territorio, aunque la base primaria del conjunto se basa mayoritariamente en centrales hidroeléctricas. Si bien, luego de la crisis del agua ocurrida en Brasil, en 2014, su modelo energético se ha expandido algo, también se destaca la necesidad de inversión y enfoque en otras fuentes de energía alternativas y complementarias.

Ante esto y ante los desafíos que plantea el escenario ante el agotamiento ambiental y el cambio climático constante, se compone un amplio modelo de incertidumbres para el tema del agua, ya que, entonces, surge la necesidad de una diversificación estratégica de la matriz, en el que este trabajo sugiere como perspectiva a considerar, la energía nuclear, en tiene destacado su potencial de manera sublime:

Sua elevada capacidade, da ordem de 90\%, traduz-se numa geração de base firme que propicia a confiança e a segurança de abastecimento necessárias para a utilização expressiva de fontes renováveis variáveis, como a solar e eólica. Ainda, importa destacar que [...] os turbogeradores das nucleares, contribuem, significativamente, para a estabilidade do sistema, compensando oscilações transitórias da rede, mantendo a frequência dentro de padrões adequados. (ALBUQUERQUE, 2020, p.31) 
La preocupación, sin embargo, se refiere a la explotación nuclear puesta en un modelo que sea capaz de superar el estigma heredado del negativismo y entrar en el uso de esta fuente como potencial creciente de los factores de transición y seguridad energética. Si bien este estigma no termina, el país se enfoca en la estrategia de expansión a través de su influencia externa, comenzando por la exportación de uranio en forma de commodities.

Sin embargo, Brasil, que ocupa la posición 09 (nueve) entre las mayores reservas de uranio del mundo, tiene mapeado solo el 30\% del territorio nacional, con una previsión de reanudar las operaciones de prospección geológica a fines de 2020. Según el Ministro de Minas e Energia, Bento Albuquerque (2020), la reanudación es la primera fase para consolidar nuestra propuesta de hacer de Brasil autosuficiente y exportador de uranio. Cabe señalar también que, según Reis (2015), Brasil, además de tener una de las mayores reservas de uranio y poseer el dominio tecnológico de todo el ciclo de enriquecimiento de esta fuente, el país tiene solo 1,9 GW de generación de energía. energía y sólo el 1,3\% de toda la matriz energética está ocupada por energía nuclear, que tiene operaciones concentradas en las plantas Angra 1 y 2, con la expectativa de ampliar esta matriz a 3,39 GW con la planta Angra 3. (ALBUQUERQUE, 2020)

Este argumento cobra validez, asumiendo que el país utiliza su influencia en la exportación de productos primarios para volverse autosuficiente y ampliar su credencial exportadora, con el objetivo de explorar mejor las fuentes disponibles y accesibles en el territorio nacional, incluyendo contribuir a la formación de precios. en el escenario internacional, dada su dimensión de riqueza - explorada y aún por explorar - de este mineral.

En este aspecto y, con base en las perspectivas de futuro del Programa Nuclear Brasileño, la fabricación de componentes que complementan el ciclo del combustible y la regulación y fortalecimiento de sus acciones, además de la autosuficiencia en la producción de radioisótopos y radiactivos. fuentes, se destaca. Por lo tanto, se revela un panorama estratégico de inversiones inversiones para incrementar la participación brasileña en el dominio del ciclo del combustible, atendiendo la demanda interna y la reanudación de las exportaciones. (DORILEO, 2020)

Cuando ampliamos el alcance de las perspectivas de la energía nuclear, comparándola con otras fuentes de energía, podemos entender el predominio de otros mercados superpuestos a la expansión de las termoeléctricas, esto sucede, básicamente porque el foco está en expandir el mercado renovable mientras, teóricamente, opera. en el desarrollo de tecnologías nucleares, eficaces y seguras que permitan esta relativa expansión. Por ello, actualmente se aplica el modelo de expansión y enfoque en "mercados asociados a la tecnología nuclear, como medicina nuclear, radioisótopos, control de plagas, irradiación de alimentos, marcadores de erosión, defensa (submarino de propulsión nuclear), desalación, generación, generación, hidrógeno, etc." (ENERGÉTICA, 2019, p. 127).

\subsection{El caso uruguayo como modelo de transición y seguridad energética}

La necesidad de producir un plan energético diversificado que converja con sus objetivos, hace que el Estado se enfoque en el mejor uso de los recursos internos y promueva la oferta de un modelo de desarrollo ascendente y cree condiciones favorables para un cambio de paradigma, incorporando nuevos medios energéticos 
para compatibilizar una evolución convergente con los nuevos retos medioambientales. Puede aclarar que el Plan Estratégico de Energía debe:

criar condições para a mudança do modelo energético atual favorecendo a maior contribuição das energias renováveis, da hídrica, da eólica, solar, biomassa, da energia nuclear, dos biocombustíveis e do hidrogênio, de forma a compatibilizar o desenvolvimento com a proteção do ambiente e a redução das emissões de gases com efeito de estufa. (SANTOS et al, 2016, p. 5)

Adicionalmente, hay que destacar la necesidad de mirar los desafíos y oportunidades a través de este plan estratégico, considerando que la elaboración de estas políticas tiene el principal alcance de evitar la exposición del Estado a riesgos y debe, necesariamente, tomar en cuenta aspectos esenciales y esencial, si no intuitiva, la conveniencia de diversificar el origen de los recursos, las fuentes de energía adquiridas, las rutas y modos de transporte, o incluso la distribución espacial de la infraestructura energética interna.

Podemos mencionar en este caso a Uruguay, que recientemente atravesó intensas inversiones para llevar a cabo el proceso de transición luego de un severo período de colapso en el sector energético, en el cual hubo varias interrupciones en el suministro energético nacional. Este proceso de desarrollo de una matriz más limpia se debió a la búsqueda uruguaya de seguridad energética.

Para ello, fueron necesarias varias etapas y desarrollos masivos incluidos en su Plan Estratégico de 'Política Energética 2005-2030'. El cual utilizó los conceptos y aspectos previamente resaltados sobre el fortalecimiento de la visión de largo plazo, proyectando un futuro predecible sobre su modelo, complementando y convergiendo con planes para implementar un nuevo marco institucional y regulatorio consistente con el establecimiento de lineamientos estratégicos, operativos y sobre todo inclusivo que satisfaga su potencial de demanda nacional. (CORREAA; MALDONADO; VAZ, 2012). O resultado de esta política de transición, basada en lograr su propio crecimiento y lograr su emancipación energética, Uruguay logró no solo el título de país con el mayor porcentaje de energía eólica generada en América América ocupando la $3^{\mathrm{a}}$ posición en el mundo, como por otro lado, invirtió el papel de un excelente comprador y se convirtió en el exportador.

En consecuencia, el modelo uruguayo puede verse como un punto de partida o incluso el cruce del puente para reducir su vulnerabilidad interna y energética, lo que ha transformado su indisponibilidad en oportunidades de evolución hacia una política energética basada en la maximización de su independencia y en la aplicación. de inversiones con nuevos parámetros en cuanto al aprovechamiento de la tecnología eólica que comprende la oferta, el proceso de transformación energética y la satisfacción de la demanda interna y externa.

En vista de esto, cuando lo miramos desde esta perspectiva, logramos una mejor visión para evaluar y comprender el potencial nuclear como cualquier otra fuente de energía - como el ejemplo del caso uruguayo y su expansión con foco en la energía eólica - que, puede ser visto como un recurso adicional y complementario a la matriz de países, en los que la preocupación en relación al uso de fuentes internas de energía va más allá del enfoque del miedo y entra en los umbrales de la seguridad energética, especialmente en lo que se refiere a su apoyo para una nueva fase de transición. 
Por eso, cuando explicamos el plan de transición uruguayo, entendemos las especificaciones de cada recurso, sin embargo, no nos limitamos a eso. Buscamos analizar y acercar el debate para observar en qué medida Brasil avanza en sus estrategias y el potencial natural que tiene internamente, que actualmente está desaprovechado por la falta de desarrollo tecnológico, falta de recursos estratégicos o incluso por un cierto 'preconcepción' debido a la falta de comprensión y al temor de un mal uso de esta fuente. De esta manera, el aporte del Plan Energético Uruguayo nos permite repensar nuevas formas de explorar internamente el contenido de nuestro verdadero potencial, que hasta ahora había estado latente.

De esta manera, vale la pena proponer un diagnóstico a partir de las lecciones aprendidas del modelo de transición uruguayo, como la articulación de instrumentos de gobernanza, además de una alianza institucional en los ámbitos ambiental, legislativo, regulatorio y regulatorio, además de posibilitar y ampliar el acceso a la información por parte de la población, para que puedan beneficiarse de este abanico de posibilidades asumido cuando tratamos al uranio como una fuente indispensable para la transición y un recurso tan expresivo como otras fuentes, incluso renovables (solar y eólica, específicamente).

\section{Algunas reflexiones finales}

Ante un mundo en constante transición, debido a las nuevas necesidades ambientales y climáticas, aquí se pretendía plantear los debates sobre el uranio, sus funcionalidades y sus desafíos en el ámbito legal y de seguridad, sin embargo, cabe destacar el descubrimiento de un nuevo conciencia sobre el potencial de este recurso, destacando que esta fuente, así como otros recursos energéticos, incluidos los renovables, incorpora en su alcance incertidumbres de transición provocadas básicamente por su proceso tecnológico y adaptación a su matriz. Sin embargo, este es considerado un recurso natural con un alto potencial de crecimiento, siendo fundamental para el proceso de desarrollo y generación de energía.

Así, creemos que es necesario para el desarrollo de este trabajo considerar no solo la agilidad, sino el proceso de transición energética brasileña, su contexto histórico y su desarrollo en el tiempo, enfocándose en el nivel de desarrollo de la energía nuclear y sus perspectivas basado en un escenario actual, regido por incertidumbres e inconsistencias importantes en diferentes áreas, ya sean económicas, sustentables o ambientales.

Con eso, nos dimos cuenta de que, si bien la transición y la seguridad energética son temas prácticamente complementarios, estos en cierto punto, se vuelven, en cierto modo, antagónicos entre sí. Es decir, al mismo tiempo que el factor ambiental es un rostro que propone una emergencia de transición, este mismo factor puede ser un punto de atención si se observa por el ángulo de (in) disponibilidad de recursos, los cuales son más limpios y accesibles además a ser sostenibles y eficientes que proporcionen la oferta en términos satisfactorios de la demanda local.

Sin embargo, la importancia de la ponderación es necesaria para que podamos realizar un análisis crítico en el que se pretenda mejorar los resultados en relación a la seguridad 
pública y energética que se colocan como el principal espectro que acecha al manejo de la energía nuclear en su conjunto.

En suma, además de los factores que se traslapan al uso de la energía nuclear, buscamos observar el plan de transición de Uruguay, descrito en el documento del Plan de Energía (PE, 2005 - 2030), que, en un corto período de tiempo, se convirtió en el gigante mundial de la transición energética, implementando decretos y resoluciones en su sistema interno que le permitirían el título de liderazgo global y, sobre todo, el rol de pionero en temas renovables. Este estudio contribuyó a una visión de buenas prácticas de conciliación, gobernanza y gestión interconectada que apalancó el proceso y tuvo éxito en esta nueva etapa de la transición energética y sobre todo en las áreas de seguridad e integración de la población y expansión del entorno empresarial.

Implementado de manera socialmente inclusiva, el plan uruguayo buscó sobre todo impulsar la innovación, que conduzca al desarrollo energético interno y la independencia del país, logrando estratégicamente su seguridad energética basada en el atractivo político, fiscal y económico, desprendiéndose de las fuentes fósiles y abandonando, en consecuencia, a la relativa volatilidad de sus precios. Con base en estos parámetros, se analizó el caso actual de Brasil, llegando a una breve conclusión que, si bien el país tiene potencial para apalancar el uso del uranio, aún existen necesidades internas por atender, como el apoyo a la inversión, la sociedad de integración y el las propias entidades gubernamentales.

En definitiva, existe un potencial de exploración en relación con Brasil desde el momento en que logra mitigar los estigmas y pensar en el recurso nuclear como una fuente complementaria que permite satisfacer la demanda interna y una menor dependencia de las fuentes de agua, garantizando así, consecuente seguridad energética.

\section{Referencias bibliográficas}

Aben. (2020). Carta aberta ao setor energético. Associação Brasileira de Energia Nuclear, 2020 (723). Recuperado de http:/ / www.aben.com.br/Arquivos/723/723.pdf

Albuquerque, B. C. L. (2020). Energia nuclear: oportunidades para o Brasil. Rio de Janeiro: Centro Brasileiro de Relações Internacionais.

Andrade, A. M. R. (2015). O programa da autonomia do ciclo de combustível nuclear no Brasil. Revista Brasileira de Pós-Graduação, Año 12 (29), pp. 609-896.

Bermann, C. (2002). Energia no Brasil: para que? Para quem? (2. ed.) São Paulo: Livraria da Física.

Braga, D. C. (2019a). Energia nuclear entre o paradigma da sustentabilidade e da transnacionalidade: possibilidades jurídicas para sua expansão na matriz elétrica brasileira no terceiro milênio. Itajaí: Univali.

Braga, D. C. (2019b). Energia nuclear e meio ambiente: princípios da precaução e da prevenção. Curitiba: Editora Appris. 
Braga, D. C. (2014). O modelo de regulação do Programa Nuclear Brasileiro e suas implicações com os princípios da precaução e da prevençãa. Manaus: Universidade do Estado do Amazonas.

Brandão, R. V. M. (2008). O negócio do século: o acordo de cooperação nuclear Brasil - Alemanha. Niterói: Universidade Federal Fluminense.

Brasil. (1969). Emenda Constitucional n. 01/1969: Edita o novo texto da Constituição Federal de 24 de janeiro de 1967. Constituição da República Federativa do Brasil. Recuperada de http://www.planalto.gov.br/ccivil_03/constituicao/Emendas/Emc_ anterior1988/emc01-69.htm

Brasil. (1988). Constituição da República Federativa do Brasil. Constituição da República Federativa do Brasil. Recuperada de http://www.planalto.gov.br/ccivil_03/constituicao/constituicao.htm

Brasil (2018). Decreto n. 9.600/2018: Consolida as diretrizes sobre a Política Nuclear Brasileira. Constituição da República Federativa do Brasil. Recuperada de http:/ /www. planalto.gov.br/ccivil_03/_ato2015-2018/2018/decreto/D9600.htm

Brasil. I. N. (2020). Brasil retoma produção de urânio. Indústrias Nucleares do Brasil, 20 (01). Recuperado de https://www.gov.br/pt-br/noticias/energia-minerais-e-combustiveis/2020/12/brasil-retoma-producao-de-uranio

Camargo, J. M. T. (2020). A Geopolítica da energia em transição. Rio de Janeiro: Centro Brasileiro de Relações Internacionais.

Corrêa, K. C.; Maldonado, M. U.; VAZ, C. R. (2012). Os desafios da transição energética das Usinas Eólicas no Uruguai. XXII ENGEMA, 22 (384). Recuperado de https:/ / engemausp.submissao.com.br/22/arquivos/384.pdf

Cruz, P. M.; Bodnar, Z.; Ferrer, G. R. (2012). Globalização, transnacionalidade e sustentabilidade. Itajaí: Editora Univali.

De Biasi, R. (1979). A energia nuclear no Brasil. Rio de Janeiro: Biblioteca do Exército.

Dorileo, I. L. (2020). Existe um lugar para a energia nuclear na transição energética (?) Agência Canal Energia, 12 (01). Recuperado de http:/ / gesel.ie.ufrj.br/app/webroot/ files/publications/12_dorileo_2020_07_28.pdf

Energética, E. P. (2019). Matriz Energética e Elétrica. ABC de Energia, 19 (01). Recuperado de http:/ / epe.gov.br/pt/abcdenergia/matriz-energetica-e-eletrica

Espiell, H. G. (1968). El Problema del Método en el Derecho Constitucional. Droit Constitutionnel et Institutions Politiques. Revista de al Facultad de Derecho y Ciencias Sociales, Año X (3), pp. 02-20.

Girotti, C. A. (1984). Estado nuclear no Brasil. São Paulo: Editora Brasiliense.

Gramkow, C.; Simões, P. B. S.; Kreimerman, R. (2019). Big Push Energético do Uruguai. Santiago: Comissão Econômica Para América Latina.

Helmreich, J. E.; Ores, G. R. (1981). The Diplomacy of Uranium Acquisition. Princeton: Princeton University Press. 
Heilbron, S. R. C. L.; Costa, S. R. R. (2017). A importância da pesquisa regulatória para um órgão regulador no setor nuclear. Revista Internacional de Ciências, Año 01 (07). Recuperado de https://www.e-publicacoes.uerj.br/ojs/index.php/ric

Honty, G. (2011). Energía nuclear en América Latina: el día después. Nueva Sociedad, Año 2011 (234), pp. 251-355.

Oiea. (2020). Climate Change and Nuclear Power. Viena: Organismo Internacional de Energía Atómica.

Presupuesto, O. P. (2019). Hacia una Estrategia Nacional de Desarrollo, Uruguay 2050 - Presente y futuro de las energías renovables en Uruguay. Montevideo: Oficina de Planeamiento y Presupuesto.

IEA. (2020). Energy security: Ensuring the uninterrupted availability of energy sources at an affordable price. International Energy Agency, 20 (01). Recuperado de https:/ / www.iea.org/areas-of-work/ensuring-energy-security

Ingulstad, M.; Lixinski, L. (2013). Raw Materials, Race, and Legal Regimes: The Development of the Principle of Permanent Sovereignty over Natural Resources in the Americas. World History Bulletin, Año 2013 (XXIX), pp. 34-39.

Lehto, J. (2017). Basics of Nuclear Phisics and of Radiation Detection and Measurement. Norway: University of Oslo.

Losekann, L.; Tavares, F. B. (2019). Política Energética no BRICS: Desafios da Transição Energética. Rio de Janeiro: Instituto de Pesquisa Econômica Aplicada.

Machado, A. D. (1980). Energia Nuclear e Sociedade. São Paulo: Paz e Terra.

Malheiros, T. (1993). Brasil - A Bomba Oculta. Rio de Janeiro: Gryphus.

Meitner, L.; Frisch, O. R. (1939). Disintegration of Uranium by Neutrons: a new type of nuclear reaction. Nature, 1939 (143). Recuperado de https:/ /www.nature.com/ nature/volumes/143/issues/3615

Neves, M. (2009). Transconstitucionalismo. São Paulo: Martins Fontes.

Nina, A. (2020). A Diplomacia brasileira e a Segurança Energética Nacional. Brasília: Fundação Alexandre Gusmão.

Nucci, G. S. (2016). Direitos Humanos versus Segurança Pública. Rio de Janeiro: Grupo Editorial Nacional.

Nunes, H. M. (2019). O Desafio está lançado: O Brasil em busca da integração energética Sul-Americana (2000-2010), Retratos Sul-americanos: perspectivas brasileiras sobre história e política externa (pp. 705-732). Brasília: Centro Universitário de Brasília.

Organization, U. N. (2019). World Commission on Environment and Development report. United Nations Organization, 20 (01). Recuperado de http://www.un.org/documents/ga/res/42/ares42-187.htm

Patti, C. (2014). O Programa Nuclear Brasileiro: uma história oral. Rio de Janeiro: Fundação Getúlio Vargas. 
Petrouzo, M. C. V. (2014). Derecho de La Energia. Montevideo: Universidad de Montevideo.

Reis, C. M. (2015). Diversificação da Matriz Energética Brasileira-Caminho para a Segurança Energética em Bases Sustentáveis. Rio de Janeiro: Centro Brasileiro de Relações Internacionais.

Santos, T. et al. (2016). MERCOSUL 2+ e Segurança Energética: Uma Análise Comparada das Interpretações do Conceito e das Políticas Energéticas Nacionais. Congresso Acadêmico sobre Defesa Nacional, 2016 (01). Recuperado de https:/ /www.gov.br/defesa/pt-br/arquivos/ensino_e_pesquisa/defesa_academia/cadn/artigos/XIII_cadnmercosul_2_e_seguranca_energetica_uma_analise_comparada_das_interpretacoes_do_conceito_e_das_politicas_energeticas_nacionais.pdf

Stoiber, C.; Baer, A.; Pelzer, N.; Tonhauser, W. (2006). Manual de Derecho Nuclear. Austria: Organismo Internacional de Energía Atómica. 